\title{
Framework for Multiple Time-Scale Cascaded MPC Application in Power Systems
}

\author{
Andreas Ulbig, Michèle Arnold, Spyros Chatzivasileiadis, Göran Andersson
}

\author{
ETH Zurich, EEH - Power Systems Laboratory \\ Physikstrasse 3, 8092 Zurich, Switzerland \\ E-Mail: \{ulbig, arnold, spyros, andersson\}@eeh.ee.ethz.ch \\ Web Site: www.eeh.ee.ethz.ch
}

\begin{abstract}
A framework for the application of cascaded Model predictive control (MPC) in power systems for controlling processes on multiple time-scales is presented. Power system control and optimisation is traditionally accomplished on time-scales ranging from milliseconds (protection systems, primary voltage and frequency control) to several years (grid expansion planning). Employing an MPC scheme for controlling or optimising power system properties over several time-scales is in general computationally prohibitive. Instead, separate MPC schemes can be implemented, each designed for one time-scale, working in parallel on the same or different tasks, acting on separate time-scales and interacting via updates of each others' constraints and cost terms. The resulting cascaded MPC scheme remains computationally tractable. Its operational principles are illustrated by simulation examples.
\end{abstract}

Keywords: Electric power systems, power system control, model predictive control, cascade control.

\section{INTRODUCTION}

\subsection{Overview of Power System Control, Operation and Planing}

Power system control and optimisation is accomplished on varying time-scales ranging from seconds to minutes in the case of voltage and frequency control, from minutes to hours in the case of intra-day and day-ahead power dispatch, from days to weeks to months for pump-storage optimisation and from months to several years in the case of capacity planning for electricity transmission, storage and generation. Furthermore, power system control structures for regulating a given system variable, for example voltage levels or frequency, are usually realised via a number of nested control loops. An illustration of the nested control-loops for grid frequency regulation and their activation pattern is given in Figure 1. Primary frequency control is accomplished by responsible generation and storage units anywhere in an inter-connected transmission system. Secondary frequency control is done only by units within the control zone in which the initial fault occurred. For calling up tertiary frequency control, the topology and the congestion status of the transmission grid within the respective control zone needs to be taken into account. Time control corrects global time deviations of the synchronous time. Since the time-scales for activation and duration of primary to tertiary frequency control range from seconds to hours, the three control loops are practically de-coupled from each other, but called to action in a cascaded fashion depending on the duration of a frequency deviation and the location of the fault within the transmission grid. This is also generally true for other power system controls, for example voltage regulation, resulting in numerous de-coupled control loops operating in parallel and on different time scales within a power system. Note that this paper refers

\footnotetext{
* Financial support from the research projects "Infrastructure Roadmap for Energy Networks in Europe" (IRENE-40) of the European Commission (EC) and "Vision of Future Energy Networks" (VoFEN) of ABB, Areva T\&D, Siemens and the Swiss Federal Office of Energy, is gratefully acknowledged.
}

to the power system conventions used in Continental Europe (ENTSO-E, 2009).

In a power dispatch optimisation, in which energy is dispatched in time blocks of individual hours (EPEX, 2010), there is no consideration of the underlying voltage and frequency control loops, since their actions are not happening on the same timescale. However, other considerations such as transmission line constraints are taken into account. In the optimisation of pumpstorage unit operation, daily effects (pumping at night-time, generating at day-time) as well as seasonal effects (dry/rainy season) may need to be considered. For the optimal planning of new transmission, generation and storage capacities, expected future load demand and expected future electricity prices are the key factors for making investment decisions.

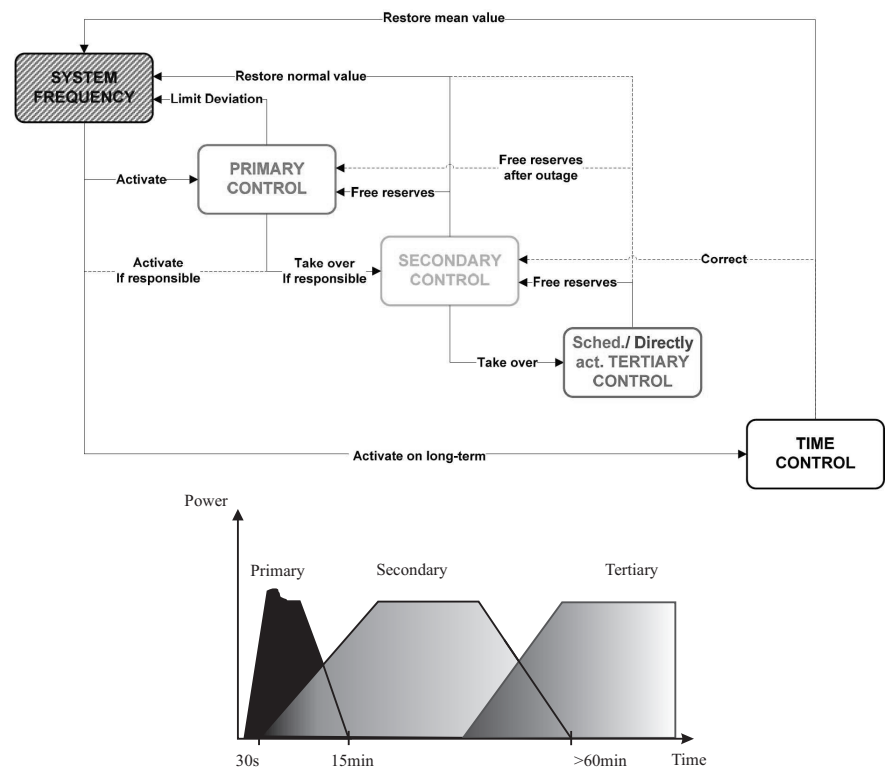

Fig. 1. a) Nested control loops for grid frequency regulation (ENTSO-E, 2009). b) Activation pattern after a fault event. 


\subsection{Process interdependencies on different time-scales}

Traditionally, power system control and operational tasks are treated separately and, often, independent from one another. For example, frequency control and power dispatch are treated as separate topics, having differing objectives (Frequency control: keeping grid frequency $f$ at nominal level $f_{0}$ - Power dispatch: reducing overall economic cost of electricity production) and different constraints (Frequency control: available power and energy reserves - Power dispatch: line transmission and power plant capacity as well as energy reserves of storage units). Nevertheless, important interactions occur between both tasks. Large-scale deployment of intermittent renewable energy sources (RES) as well as increasing power market activity are both leading to more frequent generation schedule changes and more fluctuations in transmission patterns. The resulting more frequent transient power mismatches and power flow changes in turn trigger the use of frequency regulation reserves. This has become a nuisance for grid operation (Weissbach and Welfonder, 2009). However, there is no automated feedback loop between frequency regulation and power dispatch processes. Another example is grid expansion planing. Here, investment decisions are driven by long-term trends such as load demand evolution and generation unit placement. On the one hand, power transmission and generation usage patterns within a grid depend mainly on the outcome of the power dispatch. If the dispatch results shift considerably due to changing power system economics, e.g. changes in fuel costs, introduction of a carbon price or a RES feed-in tariff, transmission and generation usage patterns will as well. This will eventually change the needs for transmission and generation infrastructure. On the other hand, building new transmission and generation capacity also alters the output of an economic dispatch optimisation. Furthermore, operation problems such as frequent line congestion or seasonally recurring events like rolling black-outs during extreme weather conditions may also trigger the decision to build new infrastructure. Such information on grid operation performance should be passed on into grid expansion planning tools.

Several interdependencies exist between control loops and optimisation processes that are acting simultaneously in power systems. They should only be perceived as decoupled processes, and hence neglected, under the assumption of steady-state grid operation. Frequent power plant operation point and power flow changes are increasingly testing such steady-state assumptions. Therefore, an integrated framework for power system control and operation on multiple time-scales is proposed that explicitly accommodates interdependencies via a cascaded control and operation scheme. MPC is used, as all relevant power system processes (frequency/voltage control, power dispatch and grid expansion planning) can be implemented using the capabilities and advantages that MPC provides: definition of a control strategy via cost functions and explicit consideration of process constraints. Communication between the individual MPC instances is realised by updating constraint and cost terms.

The paper proceeds as follows: In Section 2, the cascaded MPC framework for power systems is introduced. In Sections 3-5 the application of MPC to different power system processes (transmission expansion planning, energy dispatch and frequency control respectively) is highlighted. Section 6 presents simulation examples employing cascaded MPC schemes. Finally, Section 7 states conclusions and outlines future research.

\section{CASCADED MPC FOR POWER SYSTEMS}

\subsection{Motivation}

A cascaded MPC framework for power systems control and operation, which accommodates interdependencies between processes on different time-scales, needs the following properties:

(1) An information exchange on the interdependencies between individual processes shall be established.

(2) The computational complexity of the individual processes shall not increase.

(3) The control performance of the individual processes shall be higher than without this framework.

Individual MPC schemes for separate power system control and operation processes need to be implemented and connected with each other. Several examples for MPC application to individual power system tasks have been published in recent years (Beccuti et al., 2007; Negenborn et al., 2007; Xie and Ilić, 2009; Arnold et al., 2009; Ulbig et al., 2010). The proposed framework is a setup of nested MPC schemes arranged in the form of a cascade. Nested or cascaded MPC schemes have been proposed for other applications, notably in the chemical industry with the goal of complexity reduction and improved performance (Ying et al., 1999). However, to our knowledge, cascaded MPC schemes have not yet been proposed for accommodating interdependencies between processes on different time-scales in the context of power system control and operation. The choice to use MPC in this framework is motivated by the well-known advantages of MPC as a control and optimisation scheme over classical control methodologies, i.e. control objectives can be stated in the form of a cost function that is minimised in a receding horizon fashion, while respecting explicitly defined system state and control input constraints.

\subsection{General Formulation of an MPC Problem}

MPC is a widely accepted control methodology, combining features of both optimal and predictive control. MPC traditionally involves the iterative solution of a constrained finite-time optimal control (CFTOC) problem for the system state $\mathbf{x}(k)$ of a given plant model at each sampling step $k$ for a finite prediction horizon $T_{p}=N * k$, i.e. $\left[k, k+T_{p}\right]$. In each iteration only the first step $\mathbf{u}(k)$ of the full cost-minimizing control strategy $\left[\mathbf{u}(k), \ldots, \mathbf{u}\left(k+T_{p}\right)\right]^{T}$ is implemented. The optimisation is then repeated for the updated system state $\mathbf{x}(k+1)$ (Mayne et al., 2000). A generalised MPC setup for discrete-time linear timeinvariant (LTI) systems in state-space form is given as follows:

$$
\begin{aligned}
& \min _{\mathbf{u}(\cdot)}\left\{\sum_{k=0}^{N-1}\left(\|\mathbf{Q} \mathbf{x}(k)\|_{l}+\|\mathbf{R} \mathbf{u}(k)\|_{l}\right)+\left\|\mathbf{Q}_{\mathbf{f}} \mathbf{x}\left(k+T_{p}\right)\right\|_{l}\right\} \\
& \text { s.t. }\left\{\begin{array}{l}
\mathbf{x}(k+1)=\mathbf{A} \mathbf{x}(k)+\mathbf{B} \mathbf{u}(k), \text { with } x_{0}=\mathbf{x}(0) \\
\mathbf{y}(k)=\mathbf{C} \mathbf{x}(k)+\mathbf{D} \mathbf{u}(k)
\end{array}\right. \\
&\left\{\begin{array}{l}
\mathbf{g}^{T} \mathbf{x}(k) \leq \mathbf{0}^{T}, k=0, \ldots, N \\
\mathbf{g u}^{T} \mathbf{u}(k) \leq \mathbf{0}^{T}, k=0, \ldots, N-1
\end{array}\right. \\
&\left\{\begin{array}{l}
\mathbf{Q}=\mathbf{Q}^{\prime} \succeq 0, \mathbf{Q}_{\mathbf{f}}=\mathbf{Q}_{\mathbf{f}}{ }^{\prime} \succeq 0, \mathbf{R}=\mathbf{R}^{\prime} \succeq 0, \text { if } l=2, \\
\operatorname{rank}(\mathbf{Q})=n, \operatorname{rank}(\mathbf{R})=m, \text { if } l \in\{1, \infty\} .
\end{array}\right.
\end{aligned}
$$


In Eq. $1, \mathbf{x} \in \mathbb{R}^{n}$ is the discrete state vector, $\mathbf{u} \in \mathbb{R}^{m}$ the discrete control input vector, $\mathbf{y}$ the system output, $T_{p}=N * k$ the prediction horizon, $\mathbf{A} \in \mathbb{R}^{n \times n}$ the system matrix and $\mathbf{B} \in$ $\mathbb{R}^{n \times m}$ the control input matrix. The optimisation vector, given as $\left[\mathbf{u}(0)^{T}, \ldots, \mathbf{u}(N-1)^{T}\right]^{T} \in \mathbb{R}^{m \times N}$, consists of all decision variables for $k=0, \ldots, N-1$. Within the minimisation setup, the first term represents the stage cost and the second term the terminal state cost. These cost functions are defined via weighting matrices: $\mathbf{Q}$ is the cost term for system state $\mathbf{x}(k)$, $\mathbf{Q}_{\mathbf{f}}$ the terminal system state cost at the end of the prediction horizon $\mathbf{x}\left(k+T_{p}\right)$, and $\mathbf{R}$ the cost term for the control input $\mathbf{u}(k)$. The $q_{x}+q_{u}$ constraints imposed on $\mathbf{x}$ and $\mathbf{u}$ are defined by $\mathbf{g}_{\mathbf{x}} \in \mathbb{R}^{m \times q_{x}}$ and $\mathbf{g}_{\mathbf{u}} \in \mathbb{R}^{m \times q_{u}}$, respectively. The term $l$ in $\|\cdot\|_{l}$ specifies the chosen norm for the cost function as $l \in\{1,2, \infty\}$.

The advantages of MPC are that constraints on $\mathbf{x}$ and $\mathbf{u}$ can be handled explicitly in the design stage. Furthermore, optimal performance as well as closed-loop stability can be guaranteed through the incorporation of additional constraints into the setup (Chen and Allgöwer, 1998; Primbs, 1999; Jadbabaie, 2000; Morari and Lee, 1999; Ebenbauer et al., 2009). Decentralized and distributed MPC setups have been proposed, which are particularly advantageous for coping with the large-scale nature of electric power grids (Damoiseaux et al., 2008).

\subsection{Creating a cascaded MPC setup}

\section{Computational Complexity}

Employing a single MPC scheme for power systems control on several time-scales is, in general, computationally prohibitive. This is illustrated by a small example, in which grid frequency regulation as well as an intra-day power dispatch shall be accomplished by a single MPC scheme. On the one hand, a small sampling time $T_{s}$ for each sampling step $k$, e.g. a few seconds at most, is necessary for a sufficiently fast reaction to frequency deviations. On the other hand, a large prediction horizon $T_{p}$ is needed for a sufficiently long dispatch look-ahead, e.g. a few hours. Such a setup would lead to a computationally prohibitive high number $N$ of prediction steps $k$ from the current time step to the end of the prediction horizon, since $N=T_{p} / T_{S}$. For an MPC setup in which $T_{s}=5$ seconds and $T_{p}=4$ hours, this would result in $N=T_{p} / T_{s}=2880$ steps.

\section{Interaction between Individual MPC Entities}

Instead, two separate MPC schemes can be employed. Each setup is designed for only one time-scale, i.e. having a sampling time and prediction horizon adapted to its particular control task. The two MPC schemes work in parallel on their respective tasks (frequency regulation and power dispatch), acting on separate time-scales, and interact with each another by updating the constraints and cost terms. The boundaries for possible control action are set by the higher-level MPC process (power dispatch) and passed along to the lower-level MPC process (frequency regulation) as updated control input and system state constraints. In turn, the resulting grid control performance as well as updated constraint conditions can be passed on as additional input from the lower-level MPC process to the higher level MPC process in the form of an updated cost function term. This interaction structure results in a cascaded MPC scheme.

An extended example of our framework for cascaded MPC application to power system control processes on different timescales is illustrated in Figure 2. Here, three MPC-driven power system control processes are depicted: A transmission expansion planning process acting on a time-scale of months to years
( $T_{s}=1$ month and $T_{p}=1-10$ years), a power dispatch acting on a time-scale of hours to days $\left(T_{s}=1 \mathrm{~h}\right.$ and $\left.T_{p}=24 \mathrm{~h}\right)$ and, finally, a grid frequency regulation scheme acting on a time-scale of milliseconds to a few minutes $\left(T_{s}=20 \mathrm{~ms}\right.$ and $\left.T_{p}=1 s \ldots 15 \mathrm{~min}\right)$. These processes are presented in detail in the following sections. The interaction between the individual processes is accomplished via updates of constraint and cost terms in the respective MPC setups. In this example, two interaction relationships exist, which are explained in the following.

Interaction: Transmission Planning (TP) - Power Dispatch (PD) The MPC-driven transmission planning process can resize transmission capacities between grid nodes as well as generation and storage capacity at individual nodes. The information of a change in grid topology and generation/storage capacity can then be passed along to the MPC-driven power dispatch in the form of updated constraints for control inputs (available power from generation and storage units $\mathbf{u}_{\text {gen }}$ and $\mathbf{u}_{\text {storage }}$ ) and system states (available storage capacity $\mathbf{x}_{\text {storage }}$ ). Such constraint updates will eventually alter the results of the power dispatch process. The updated dispatch results can then be passed back to the transmission planning process in the form of a welfare function, indicating the dispatch's performance for the rearranged transmission grid setup.

$\begin{aligned} \text { TP deployment: }\left(u, x_{S O C}, L_{\text {line }}\right)_{i} & \Rightarrow \text { PD update: }\left(u, x_{S O C}\right)_{i} \\ \text { PD upd. dispatch: }\left(u, x_{S O C}\right)_{i} & \Rightarrow \text { TP: welfare function of PD }\end{aligned}$

Interaction: Power Dispatch (PD) - Frequency Regulation (FR) The MPC-driven power dispatch defines the generator and storage unit set-points for any given hour of the next day based on economic considerations and the given grid transmission constraints. The dispatch process thus sets the operation constraints for the MPC-driven frequency regulation process, i.e. which conventional generators will be running at what output level for a given hour and what will be the state-of-charge (SOC), $\mathbf{x}_{\text {storage }}$, of the storage units. These are direct operation constraints for grid frequency regulation as spinning generators, $\mathbf{u}_{\text {gen }}$, and storage units, $\mathbf{u}_{\text {storage }}$, are its principal power reserves for control actions. The frequency regulating MPC setup will check whether or not positive and negative regulation reserves will be sufficient for every given hourly time-step $k$ of the power dispatch process. If this is not the case, a demand to adjust generator set-points will be fed-back into the dispatch process. Additionally, the performance of the frequency regulation process is given as a feedback for the power dispatch of the next hourly time-step in the form of a cost term that drives the dispatch set points into a more stable but potentially less economic range. This performance measure includes effects from frequency disturbances caused by fluctuating renewable energy sources (RES) or mismatching generator ramp rates, e.g. a gas-turbine quickly ramping down at the end of a dispatch interval combined with a coal-fired station slowly ramping up at the beginning of the next dispatch interval.

\section{PD choice: $\left(u, x_{S O C}\right)_{i} \Rightarrow$ Availability for FR: $\left(u, x_{S O C}\right)_{i}$ \\ FR activation: $\left(u, x_{S O C}\right)_{i} \Rightarrow$ PD storage update: $x_{S O C_{i}}$}

The general idea of our framework, to explicitly define interdependencies and interactions between various MPC-driven subtasks, can be extended to also include other relevant power system processes like grid voltage regulation or coupled dayahead and intra-day power dispatch schemes. 
Please note that not all constraints are valid on all time-scales, e.g. transmission line limit constraints, Eq. 2, are important for a power dispatch process on the time-scale of minutes to hours but essentially meaningless for a frequency regulation process on a time-scale of seconds to only a few minutes. In return, a power unit's maximum ramp rate constraints are of more relevance for frequency regulation than for power dispatch.

This is in turn yet another advantage of the here proposed cascaded MPC framework. Existing constraints are only implemented for the processes for which they are of relevance, yet the information of a constraint's existence can implicitly be passed on to lower-level or higher-level processes.

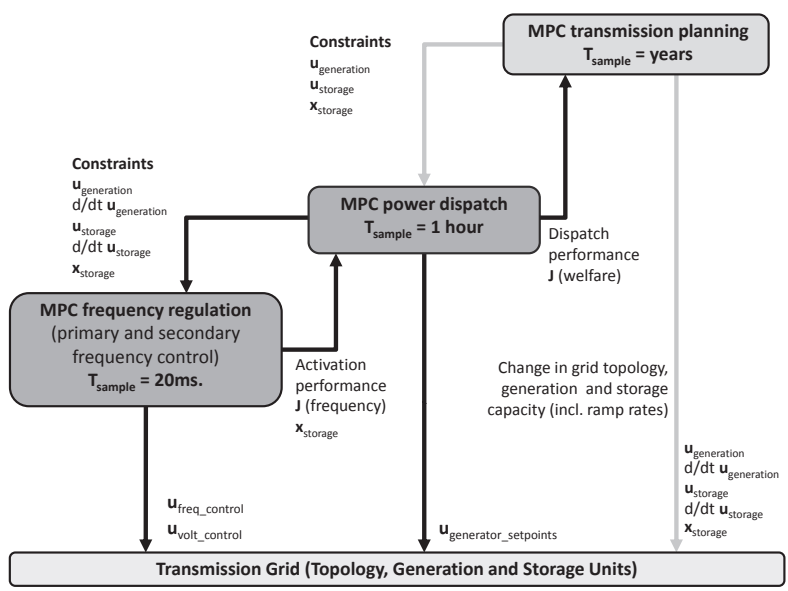

Fig. 2. Cascading MPC framework for Power Systems.

\section{MPC FOR TRANSMISSION GRID PLANNING}

\subsection{Motivation}

As already mentioned, transmission grid planning deals with operation of new lines and the connection of new equipment such as flexible AC transmission devices and storage. The stochastic power in-feed from many renewable energy sources, which were widely deployed the last decade (REN21, 2010), allows only a limited contribution to the secure and reliable operation of power systems. Due to the stochastic nature of the power in-feed from many renewable energy sources, they only provide a limited contribution to the secure and reliable operation of power systems. By themselves, intermittent RES are unable to guarantee the provision of control reserve power for a given time period. However, the combination of storage capacity with intermittent RES units is an effective means for offering control reserve power. Furthermore, uncertainties in the predicted energy generation from wind or solar power can be balanced. Storage operators may also take advantage of peak/off-peak price arbitrage, charging electricity when prices are low and discharging when prices are high.

In order to better illustrate the proposed method, the example of an off-shore wind park with additional electrical storage capacity, which should be connected to the grid through an HVDC cable will be used, see Fig. 3. Previous research efforts have examined the impacts of storage to social welfare (Sioshansi, 2010), suggested techniques for the optimal size of hydro-pumped storage in Castronuovo and Peças-Lopes (2004) and have concluded that the integration of storage may have economic benefits for the power producer (Voller et al., 2009; Oudalov et al., 2007). An MPC-based formulation seems to be the appropriate approach for the problem of optimally sizing the battery storage and the capacity of the transmission line connecting the off-shore wind park to the grid. The optimal capacities are determined by optimising the battery operation over a longer period, while considering the battery capacity and line thermal limit as slack variables.

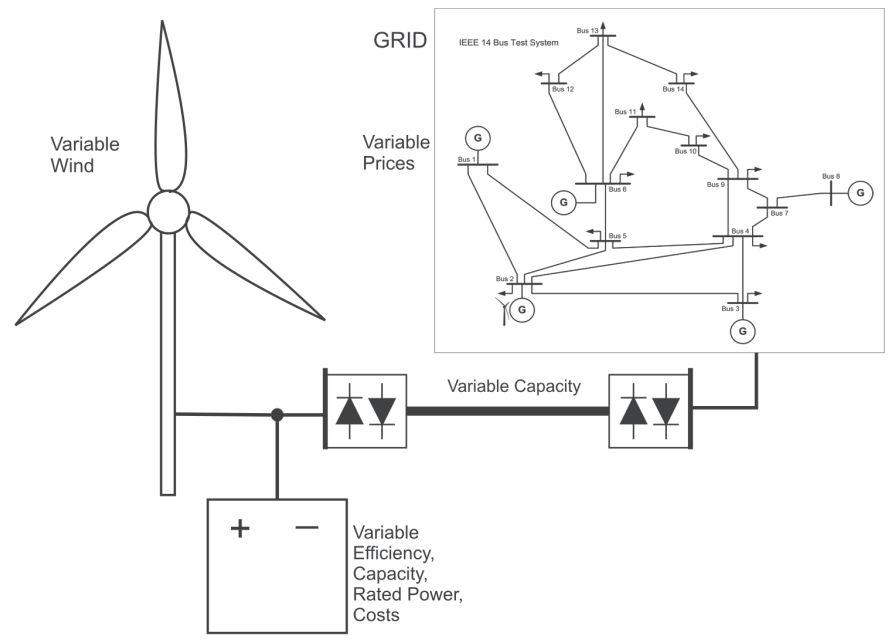

Fig. 3. Illustration of the studied power system: off-shore wind park with battery storage and HVDC link to mainland grid.

\subsection{Formulation and Optimisation Setup}

As several technologies for battery energy storage systems have been developed, batteries based on different technologies usually have different efficiency rates and different costs. In order to take the different battery technologies into account, a methodology inspired from MPC techniques, is proposed. This technique, shown in Fig. 4, alternates between an optimisation algorithm, which determines the optimal capacity of the battery, and a given set of battery models. Through the optimisation algorithm, the capacity and rated power of the battery are determined, taking into account the battery efficiency rate and the battery cost. These values are given as inputs to the battery model block, where the most suitable battery technology is selected for the defined rated power and capacity. The efficiency rate and the cost corresponding to the selected technology are then passed again as input into the optimisation algorithm and a new iteration starts. Convergence is achieved, when the efficiency rate and cost, which were used in the optimisation, correspond to the most suitable technology for the optimal rated power and storage capacity determined previously.

The optimisation algorithm is formulated as follows:

$\min _{\mathbf{u}(\cdot)}\left\{c_{\text {bat }} \cdot Q_{\text {max }}+c_{\text {line }} \cdot L_{\text {line }}+c_{B P} \cdot B P-\sum_{k=0}^{N-1} p(k) \cdot E_{\text {arb }}(k)\right\}$

s.t. $\left\{\begin{array}{l}q(k+1)=q(k)+s \cdot \eta \cdot e s(k)+(1-s) \cdot \frac{1}{\eta} \cdot e s(k), \\ \text { where } s=\left\{\begin{array}{l}1 \text { if }(e s(k) \geq 0), \\ 0 \text { otherwise. }\end{array}\right. \\ E_{a r b}(k)=w(k)-e s(k)\end{array}\right.$

and 


$$
\left\{\begin{aligned}
0 & \leq q(k) \leq Q_{\max } \\
q(k)+\eta \cdot e s(k) & \leq Q_{\max } \\
0 & \leq q(k)+(1 / \eta) \cdot e s(k) \\
-B P & \leq \dot{e s}(k) \leq B P \\
0 & \leq w(k)-e s(k) \leq L_{\text {line }} \\
0 & \leq w(k) \leq W_{\max }(k) \\
0 & \leq Q_{\text {max }} \\
0 & \leq L_{\text {line }}
\end{aligned}\right.
$$

\section{MPC FOR POWER DISPATCH IN ENERGY HUBS}

(d)

(e)

\subsection{Motivation}

(f) Due to decentralized generation (DG) technologies, such as PV installations, wind turbines, micro combined heat and power

$(g)$ plants $(\mu \mathrm{CHP})$, biomass-fired plants and others, energy produc-

(h) tion is transformed from a paradigm of a few centralized units (i) to numerous and technically diverse distributed units, located at

(i) lower voltage levels (Chicco and Mancarella, 2006). Moreover,

$(k)$ the intermittency of local RES, e.g. wind and solar power, as well as the uncertainties in their predicted available power output, create the need for storage solutions and appropriate operation strategies. Another changing aspect within the current power system structure is that energy supply is no longer based only on electricity networks, but other energy carrier networks, such as natural gas, hydrogen or heating networks. These must be taken into account as well. For example, $\mu \mathrm{CHP}$ units produce simultaneously electricity and heat by consuming natural gas, therefore coupling electricity, natural gas and heat networks. Due to the availability of the various energy carriers, consumers are more flexible in their purchasing choice, which allows them to make decisions depending on criteria such as costs, reliability and system emissions. For investigating all above discussed aspects, the energy hub modelling framework is used, which takes into account multiple energy carries as well as $\mu$ CHP units, energy storage systems and local renewable energy sources. Energy systems are considered to consist of a number of interconnected energy hubs, which represent the interface between consumers and the power supply infrastructures of the different energy systems. In order to operate the storage devices in an optimal way, an MPC approach is applied, whereby unexpected fluctuations of energy prices, load demand profiles and RES input profiles can be dealt with appropriately.

\subsection{Formulation and Optimisation Setup}

The studied system consists of three energy hubs interconnected by an electricity (solid) and natural gas (dashed) network, as illustrated in Fig. 5. Each hub represents a general consumer, e.g. an aggregation of households. Each of the hubs has its own local electrical energy production $\left(\mathrm{G}_{i}\right.$, with electric power production $P_{\mathrm{e}, i}^{\mathrm{G}}$, for $\left.i \in\{1,2,3\}\right)$. Hubs $\mathrm{H}_{1}$ and $\mathrm{H}_{2}$ have access to adjacent natural gas networks $\mathrm{N}_{1}, \mathrm{~N}_{2}$. Each hub consumes electric power $P_{\mathrm{e}, i}^{\mathrm{H}}$ and gas $P_{\mathrm{g}, i}^{\mathrm{H}}$, and supplies energy to its electric load $L_{\mathrm{e}, i}$ and its heat load $L_{\mathrm{h}, i}$. For energy conversion, the hubs contain a $\mu \mathrm{CHP}\left(\eta_{\mathrm{ge}, i}^{\mathrm{CHP}}, \eta_{\mathrm{gh}, i}^{\mathrm{CHP}}\right.$ denote gas-electric and gas-heat efficiencies) and a furnace (with efficiency $\eta_{\mathrm{gh}, i}^{\mathrm{F}}$ ). The dispatch factor $v_{\mathrm{g}, i}\left(0 \leq v_{\mathrm{g}, i} \leq 1\right)$ determines how the gas is divided between the $\mu \mathrm{CHP}$ and the furnace. For each hub $\mathrm{H}_{i}$, the outputs $\mathbf{L}_{\mathbf{i}}+\mathbf{M}_{\mathbf{i}}$ and inputs $\mathbf{P}_{\mathbf{i}}$ correlate as follows:

$$
\underbrace{\left[\begin{array}{c}
L_{\mathrm{e}, i}+M_{\mathrm{e}, i} \\
L_{\mathrm{h}, i}+M_{\mathrm{h}, i}
\end{array}\right]}_{\mathbf{L}_{\mathbf{i}}+\mathbf{M}_{\mathbf{i}}}=\underbrace{\left[\begin{array}{cc}
1 & v_{\mathrm{g}, i} \eta_{\mathrm{ge}, i}^{\mathrm{CHP}} \\
0 & v_{\mathrm{g}, i} \eta_{\mathrm{gh}, i}^{\mathrm{CHP}}+\left(1-v_{\mathrm{g}, i}\right) \eta_{\mathrm{gh}, i}^{\mathrm{F}}
\end{array}\right]}_{\mathbf{C}_{\mathbf{i}}} \underbrace{\left[\begin{array}{c}
P_{\mathrm{e}, i}^{\mathrm{H}} \\
P_{\mathrm{g}, i}^{\mathrm{H}}
\end{array}\right]}_{\mathbf{P}_{\mathbf{i}}}
$$

Each hub contains both an electrical and heat storage unit. The storage devices are modelled as an ideal storage in combination with a storage interface $\left(e_{\mathrm{e} / \mathrm{h}, i}\right)$. The power exchange $M_{\mathrm{e} / \mathrm{h}, i}(k)$ at time step $k$ is defined as the difference between the actually stored energy $E_{\mathrm{e} / \mathrm{h}, i}(k)$ at two consecutive time steps, plus some

Fig. 4. Method for determining the optimal battery size. 


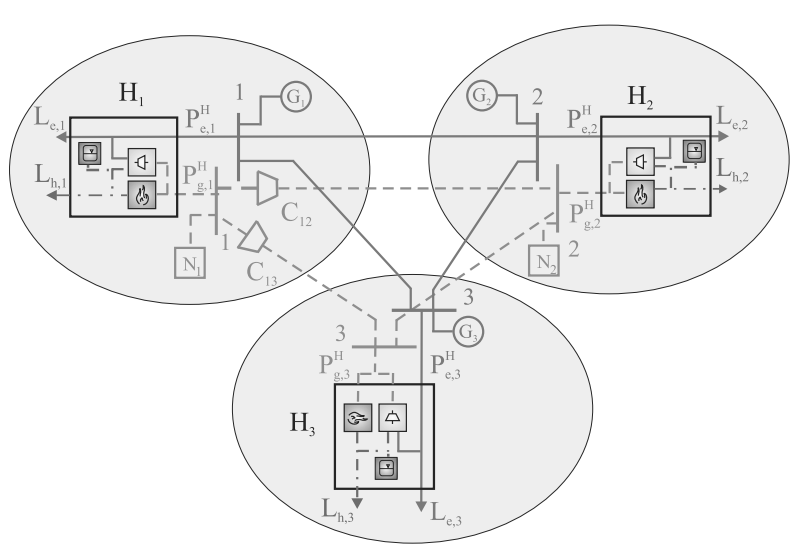

Fig. 5. System setup of three interconnected energy hubs.

stand-by energy losses $\dot{E}_{\mathrm{e} / \mathrm{h}, i}^{\mathrm{stb}}$. More detailed information on hub modelling is given in Arnold et al. (2009).

$$
M_{\mathrm{e} / \mathrm{h}, i}(k)=\frac{1}{e_{\mathrm{e} / \mathrm{h}, i}}\left(\frac{E_{\mathrm{e} / \mathrm{h}, i}(k)-E_{\mathrm{e} / \mathrm{h}, i}(k-1)}{\Delta t}+\dot{E}_{\mathrm{e} / \mathrm{h}, i}^{\mathrm{stb}}\right)
$$

The goal of the controller is to determine control variables $\mathbf{u}(k)$ in such a way that the overall operational costs of the given system are minimized while satisfying all system constraints. The main control objective is to minimize the energy costs for electricity and natural gas consumption:

$$
\begin{gathered}
\min _{\mathbf{u}(\cdot)}\left\{\sum_{l=0}^{N-1} \sum_{i_{G} \in G} q_{i_{G}}^{\mathrm{G}}(k+l)\left(P_{\mathrm{e}, i_{G}}^{\mathrm{G}}(k+l)\right)^{2}+q_{i_{G}}^{\mathrm{N}}(k+l) P_{\mathrm{g}, i_{G}}^{\mathrm{G}}(k+l)\right. \\
\left.+c_{\mathrm{Res}}\left(d^{\operatorname{Res}}(k+l)-d_{\mathrm{req}}^{\text {Res }}(k+l)\right)^{2}\right\}
\end{gathered}
$$

by determining the optimal operational set-points of the system:

$$
\mathbf{u}(k)=\left[\left(\mathbf{P}_{\mathrm{e}}^{\mathrm{G}}\right)^{\mathrm{T}}(k)\left(\mathbf{P}_{\mathrm{g}}^{\mathrm{G}}\right)^{\mathrm{T}}(k) v_{\mathrm{g}}^{\mathrm{T}}(k)\right]^{\mathrm{T}},
$$

i.e. active power generation of all generators, natural gas imports of gas networks, and dispatch factors of hubs. Thereby, the following system constraints have to be fulfilled: Equalities are the power flow equations of the transmission networks (electricity and gas) and the hub equations. Inequalities are defined by limitations on power flows, voltages, pressures, compressor settings, $\mu \mathrm{CHP}$ and furnace set-points, storage flows, etc.

Within Eq. (5), besides the minimization of overall energy consumption, the last term penalizes the deviation from the required control reserves. Control reserves are provided by the three electric storage devices and the three electric generators and are summed up in a variable $d^{\text {Res }}$. The required control reserves are defined by the load frequency regulation part, denoted by $d_{\text {req }}^{R e s}$. The higher the value of $c_{\text {Res }}$ is chosen, the more the required control reserves are fulfilled. However, operation costs may increase due to less optimal dispatching of the generation units. There is a trade-off between providing maximal control reserves and operating the system with the lowest possible operation costs.

\section{MPC FOR GRID FREQUENCY REGULATION}

\subsection{Motivation}

Regulation grid frequency $f$ is an important real-time control task and ancillary service in power systems, since deviations from nominal frequency $f_{0}$ can rapidly lead to load shedding and even black-outs. Additional control reserve capacity offers the possibility to increase the transmission grid's stability reserve margins, meaning its capability to robustly respond to occurring fault events. This can facilitate the integration of increasing amounts of fluctuating RES power in-feed.

The concept of aggregators has been proposed for the aggregation of small distributed, but controllable, generators, storage and load units in order to perform various services such as frequency regulation for power systems (Quinn et al., 2009). The idea of using plug-in hybrid electric vehicles (PHEVs) and curtailable RES generators to perform ancillary services is popular (EcoGrid, 2009; Galus et al., 2010). However, comparably small and distributed energy generation and storage units cannot contribute easily to procuring the services, as their individual power and/or storage ratings are small. Many small units have a time-variant availability, e.g. wind turbines can only be curtailed when it's windy and PHEV batteries can only balance fluctuations when connected to the grid.

An ancillary service manager is proposed, driven by an MPC setup, that allows both the management of control reserve capacity and its utilisation for grid control purposes on different time-scales. It can aggregate control reserve capacity from both fully-dispatchable sources, e.g. conventional generators, as well as from time-variant and only partially controllable sources, e.g. PHEV fleets, DSM schemes, curtailable wind generators and PV installations. For the effectiveness of this ancillary service manager, both its communication structure with underlying aggregating entities and the employed MPC setup are key factors. The potential communication structure is depicted in Fig. 6. The blocks shown on the grid level symbolise conventional generators and storages (larger blocks), wind farms (mid-sized blocks) and PHEVs as well as DSM-controlled consumers (small/mid-sized blocks). The manager communicates directly with conventional generation/storage units and indirectly via aggregator entities with distributed, smaller generation/storage units. The manager receives information on unit availability and technical constraints, which is used for updates of the MPC setup. More details are given in Ulbig et al. (2010).

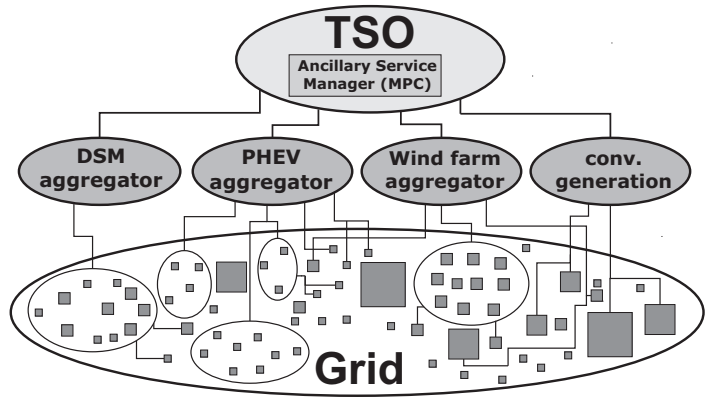

Fig. 6. Communication structure of ancillary service manager to lower level controllers.

MPC's predictive notion is of advantage here, as it allows to explicitly take into account the time-varying availability of some of the energy sources for control reserve power, e.g. 
power from wind turbines or photovoltaic (PV) installations, by using information of their predicted future power in-feed. Hence, a control reserve usage strategy can be decided upon and implemented starting at a time instant $k$, while already having in mind the predicted future availability of all types of control reserve units until the end of the finite time horizon $T_{p}$, with $T_{p}=N * k$. The manager directly implements frequency control with the aggregated power reserves while optimally dispatching them from his given portfolio of available capacity according to the grid's needs. Thereby, the manager minimizes usage costs for the control actuators, while at the same time respecting technical constraints such as ramp rates and time-variant availability of some actuators. Both tasks, aggregation and grid control, are defined, in a first stage, via a conventional MPC setup that can be solved on-line. The actual implementation is then, in a second stage, realised in the form of an explicit MPC scheme that is pre-computed off-line. Therefore, the employed explicit MPC scheme can react in real-time, e.g. $T_{s} \ll 1 s$, to occurring grid disturbances.

MPC in its traditional form has a drawback originating from its, in general, time-consuming on-line solution of the optimisation problem. However, small sampling times are necessary for the control of processes with fast dynamics like grid frequency control. This limitation of conventional MPC has motivated the search for methods that can pre-compute an optimal control law over all feasible system states. Through this process, most of the computational burden is shifted off-line. Such methods have been presented for linear systems as well as linear hybrid systems in piecewise affine (PWA) form, for both linear and quadratic cost functions (Borrelli et al., 2005; Borrelli, 2004).

\subsection{Implementation of the Ancillary Service Manager}

The implementation of the proposed ancillary service manager is realised as follows. The plant model that is given as an internal model to the MPC setup is the classical linearized swing equation in its aggregated form

$$
\Delta \dot{f}=\frac{f_{0}}{2 H S_{B}}\left(\Delta P_{m}-\Delta P_{\text {load }}\right)-\frac{f_{0}}{2 H S_{B} D_{\text {load }}} \Delta f,
$$

where $f$ is the centre of inertia grid frequency, $H$ the total inertia constant of all generators of a given grid model, $S_{B}$ the total rating of the generators, $P_{m}$ the total mechanical power of the generators and $P_{\text {load }}$ the total system load of the grid. $D_{\text {load }}$ describes part of the frequency dependency of the system load, i.e. the load's self-regulating effect (Andersson, 2010).

By using the reformulation $x_{f}:=\Delta f, x_{f}(0):=f_{0}$ and $u:=$ $\left(\Delta P_{m}-\Delta P_{\text {load }}\right)$, Equation (7) can be rephrased as

$$
\dot{x}_{f}=\frac{x_{f}(0)}{2 H S_{B}} u-\frac{x_{f}(0)}{2 H S_{B} D_{\text {load }}} x_{f} .
$$

The full system state vector $\mathbf{x}$ and the full control actuator vector $\mathbf{u}$ of the ancillary service manager are thus given as

$$
\begin{aligned}
\mathbf{x}(k)= & {\left[x_{f}(k), x_{\text {SOC }_{1}}(k), \ldots, x_{\text {SOC }_{r}}(k)\right]^{T} } \\
\mathbf{u}(k)= & {\left[u_{\text {storage }_{1}}(k), \ldots, u_{\text {storage }_{r}}(k), u_{\text {conv }_{1}}(k), \ldots,\right.} \\
& \left.u_{\text {conv }_{s}}(k), \ldots, u_{R E S_{1}}(k), \ldots, u_{R E S_{t}}(k)\right] .^{T}
\end{aligned}
$$

All constraints, both time-invariant and time-variant, for the primary system state, i.e. grid frequency, as well as for control actuators can be posed in the following uniform way:
System state (grid frequency)

$-0.5 \mathrm{~Hz}=x_{f}^{\min } \leq x_{f} \leq x_{f}^{\max }=+0.5 \mathrm{~Hz}$

Power control actuators (rated power and ramps)

$$
\begin{aligned}
& u_{\text {conv }}^{\text {min. }} \leq u_{\text {conv }_{i}} \leq u_{\text {convi }}^{\text {max. }} \\
& \dot{u}_{\text {conv }_{i}}^{\text {min. }} \leq \dot{u}_{\text {conv }_{i}} \leq \dot{u}_{\text {conv }_{i}}^{\text {max. }} \\
& \forall i=1, \ldots, s \\
& u_{R E S_{i}}^{\min }(k) \leq u_{R E S_{i}} \leq u_{R E S_{i}}^{\max }(k) \\
& \dot{u}_{R E S_{i}}^{\min }(k) \leq \dot{u}_{R E S_{i}} \leq \dot{u}_{R E S_{i}}^{\max }(k) \\
& \forall i=1, \ldots, t \text {, }
\end{aligned}
$$

Storage control actuators (rated power, energy, ramps)

$$
\begin{aligned}
& u_{\text {storage }_{i}}^{\text {min. }}(k) \leq u_{\text {storage }_{i}} \leq u_{\text {storage }_{i}}^{\text {max. }}(k) \\
& \dot{u}_{\text {storage }_{i}}^{\text {min. }}(k) \leq \dot{u}_{\text {storage }_{i}} \leq \dot{u}_{\text {storage }_{i}}^{\text {max. }}(k) \\
& 0.20 \mathrm{pu}=x_{\mathrm{SOC}_{i}}^{\min } \leq x_{\mathrm{SOC}_{i}} \leq x_{\text {SOC }_{i}}^{\text {max }}(k)=1.0 \mathrm{pu} \\
& \forall i=1, \ldots, r \text {. }
\end{aligned}
$$

where Eq. 11(a) sets a safety limit for the maximal allowable frequency deviation, $x_{f}:=\Delta f$. Equations $11(\mathrm{~b}+\mathrm{d})$ set the permissible operation range of the power rating, while $11(\mathrm{c}+\mathrm{e})$ define the permissible power ramp rates of all $s$ conventional (time-invariant) and all $t$ RES (time-invariant) units, respectively. All control actuator constraints of the $r$ storage units are defined as being time-variant: Eq. 11(f) sets the permissible operation range of the power rating, while $11(\mathrm{~g})$ is defining the permissible power ramp rates. Eq. 11(h) sets the permissible operation range for the aggregated state-of-charge, $x_{S O C_{i}}$, of the $r$ available storage units. The minimisation setup is given as

$$
\min _{\mathbf{u}(\cdot)}\left\{\sum_{k=0}^{N-1}\left(\|\mathbf{Q} \mathbf{x}(k)\|_{2}+\left\|\mathbf{R}_{\delta} \Delta \mathbf{u}(k)\right\|_{2}\right)\right\},
$$

where $\mathbf{Q}$ and $\mathbf{R}_{\delta}$ penalise deviations of the frequency $x_{f}(k)$ and set-point changes of generation/storage units $\mathbf{u}(k)$.

\section{SIMULATION EXAMPLES}

\subsection{Generation Dispatch and Frequency Control}

A case study is presented in which the proposed cascaded MPC scheme is applied to the three-hub system (Fig. 5). Interactions between the MPC-driven power dispatch and frequency regulation processes are shown. Here, the solvers fmincon (The Mathworks, 2008) and snopt (Gill et al., 2002) were used. For each hub, the daily profiles of the load demands and the energy prices are known in advance (perfect information is assumed). Based on these profiles an optimal power dispatch is done respecting the storage sizes defined by the transmission planning layer and respecting the required control reserves defined by the frequency regulation layer. Profiles and further simulation parameters can be found in Arnold et al. (2009).

For this example, a prediction horizon length of $T_{p}=3 \mathrm{~h}$ and a sampling time of $T_{s}=1 \mathrm{~h}$ have been chosen, providing a good trade-off between control performance and computational effort. The weight factor $c_{\text {Res }}=10^{3}$, ensures that control reserve availability has the highest priority. Figures 7 and 8 compare the active power generation and storage contents with and without interaction with the frequency regulation process. The positive control reserves are not satisfied when performing an optimal 
dispatch without interaction with the lower-level process (solid lines (Pg OD, E OD)). Figure 9 shows the provided positive control reserves from the optimal dispatch without process interaction ( $\left.d^{u p} \mathrm{OD}\right)$. Several times, the required control reserves $\left(d_{r e q}^{u p}\right)$ are not met (solid line). When respecting the control reserves during the simulation, generator and storage device set-points are lowered (dashed lines $\left(P_{g}\right.$ Res, E Res)). As seen in Fig. 9, the required control reserves are now met. The missing control reserves at time steps $k=10$ and $k=19$ are balanced by reducing storage in-feed and generator out-feed set-points. In order to compensate the set-point deviation from the optimal dispatch $\left(E^{u p} \mathrm{OD}\right)$, control reserves at time steps $k=14 \ldots 17$ are, in turn, reduced to the required amount. Overall, missing control reserves could be reduced from 11.9872 p.u. to 0.0237 p.u, while operational costs rose only slightly from $2.8235 \cdot 10^{4}$ m.u. (monetary units) to $2.8262 \cdot 10^{4}$ m.u. (+ $0.09 \%$ ).

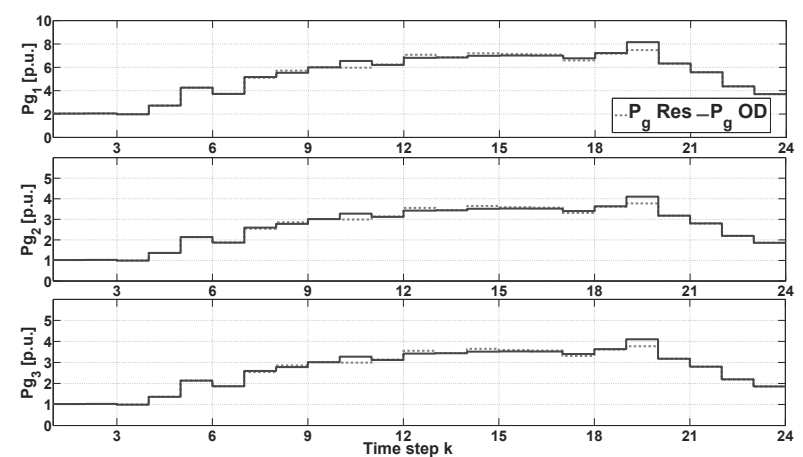

Fig. 7. Active power generation of the three generators without $\left(P_{g} \mathrm{OD}\right)$ and with $\left(P_{g} \mathrm{Res}\right)$ respecting control reserves.

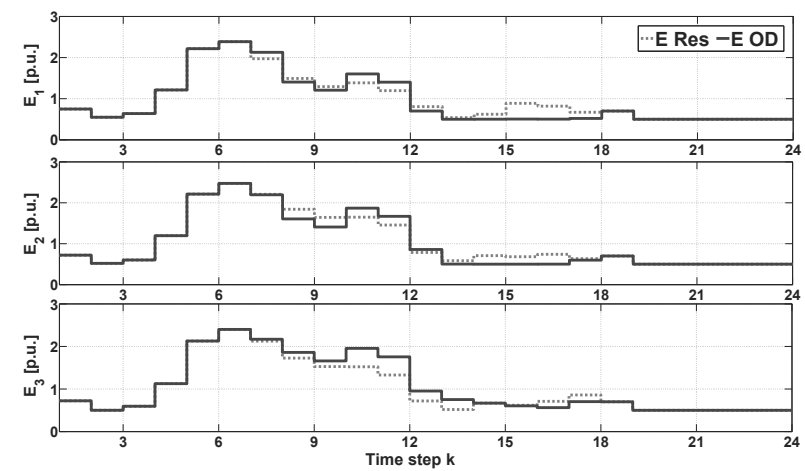

Fig. 8. State-of-charge of the three electric storage devices without (E OD) and with (E Res) respecting control reserves.

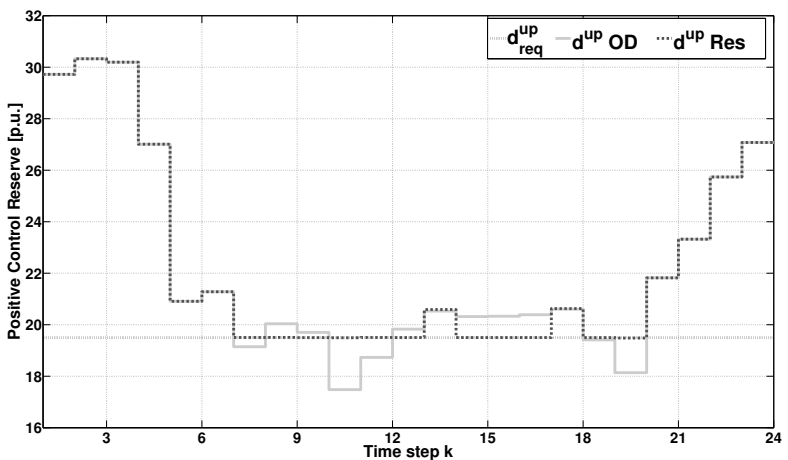

Fig. 9. Positive control reserves with ( $\left.d^{u p} \mathrm{OD}\right)$ and without ( $d^{u p}$ Res) respecting the required control reserves $\left(d_{r e q}^{u p}\right)$.

\subsection{Transmission Planning Optimisation}

The transmission planning optimisation is presented for an exemplary case. The simulation length is four days. Input data for wind power generation and electricity spot prices, based on real data for varying situations (weekday/weekend with high/low wind power in-feed and high/low spot prices), is fed into an optimal power dispatch. Typical investment costs for power/energy ratings of battery unit and transmission line are assumed (Battery: $357 \mathrm{kEUR} / \mathrm{MW}$ rated power and 74 kEUR/MWh storage capacity - HVDC line: 340 kEUR/MW rated power). Based on this, operation costs per day are calculated for an amortization period of 20 years and a discount rate of $2 \%$. The optimisation uses the solvers linprog and fmincon (The Mathworks, 2008). In the first stage, assuming an ideal battery efficiency, $\eta=100 \%$, the line thermal limit and battery unit's capacity and rated power are determined as 145.35 MW, 32.01 MWh and 5.36 MW, respectively, (Fig. 10). In the second stage, the most suitable battery technology for the specific rated power and storage capacity is found, and its efficiency rate is passed on as an input to the optimisation. For the specified ratings, the battery has a one-way efficiency of $\eta=88 \%$. The optimisation is repeated, recalculating the optimal values for the HVDC line and the battery unit, which are now 146.13 MW, 4 MWh and 0.76 MW, respectively (Fig. 11).

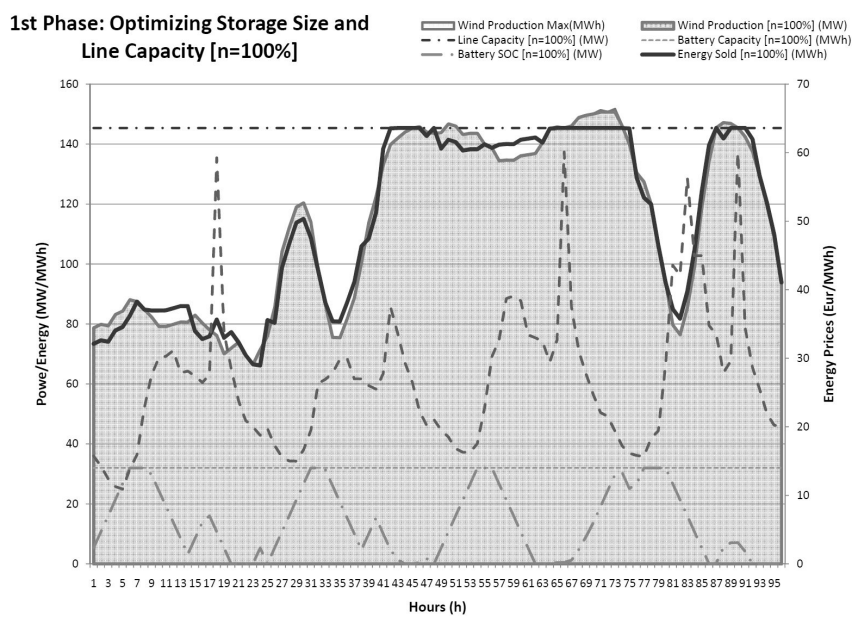

Fig. 10. Optimal system operation with pre-determined optimal battery and HVDC converter sizing (battery: $\eta=100 \%$ ).

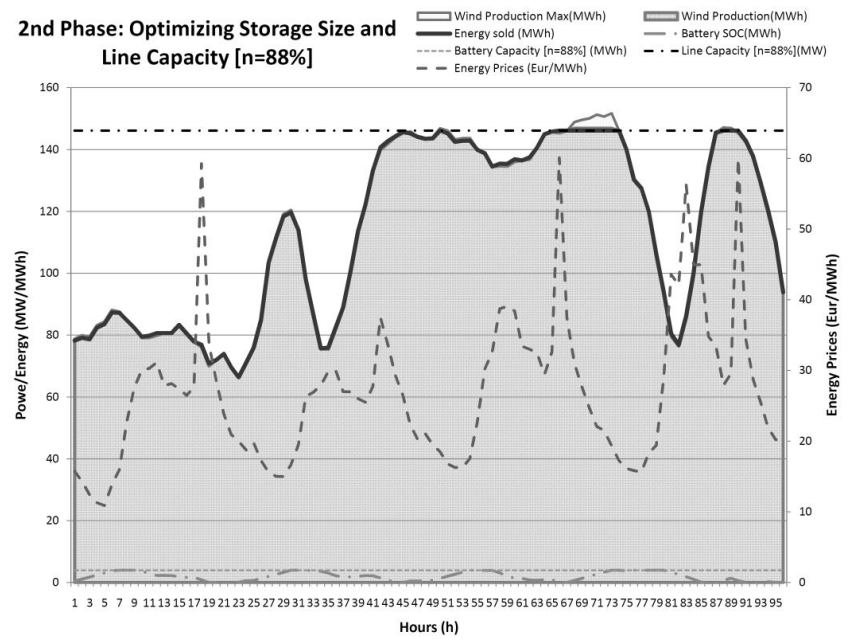

Fig. 11. Optimal system operation with pre-determined optimal battery and HVDC converter sizing (battery: $\eta=88 \%$ ). 


\section{CONCLUSION AND OUTLOOK}

A framework for the application of cascaded MPC in power systems for control and operation processes of multiple timescales was presented. The framework allows to capture interdependencies between power system control and operation processes acting on different time-scales. Simulation examples have been presented for the illustration of the concept. Future research work includes the modelling of other relevant power system processes into the framework, e.g. voltage regulation, inertia mimicking and coupled day-ahead and intra-day optimal power dispatch. This will be accomplished by taking advantage of the recently developed Power Nodes modelling framework for power systems, presented in Heussen et al. (2010).

\section{REFERENCES}

Andersson, G. (2010). Dynamics and control of electric power systems. Lecture Script, EEH-Power Systems Laboratory, ETH Zurich.

Arnold, M., Negenborn, R.R., Andersson, G., and De Schutter, B. (2009). Model-based predictive control applied to multicarrier energy systems. Presented at the IEEE PES General Meeting 2009, Calgary, Canada, 2009.

Beccuti, A., Demiray, T., Zima, M., Andersson, G., and Morari, M. (2007). Comparative assessment of prediction models in voltage control. In PowerTech Conference. Lausanne, Switzerland.

Borrelli, F. (2004). Constrained optimal control of linear and hybrid systems. Lecture Notes in Control and Information Sciences, vol. 290.

Borrelli, F., Baotić, M., Bemporad, A., and Morari, M. (2005). Dynamic programming for constrained optimal control of discrete-time hybrid systems. Automatica, 41, 1709-1721.

Castronuovo, E.D. and Peças-Lopes, J.A. (2004). Optimal operation and hydro storage sizing of a wind-hydro power plant. International Journal of Electrical Power \& Energy Systems, 26(10), 771 - 778.

Chen, H. and Allgöwer, F. (1998). A quasi-infinite horizon nonlinear model predictive control scheme with guaranteed stability. Automatica, 34(10), 1205-1218.

Chicco, G. and Mancarella, P. (2006). A comprehensive approach to the characterization of trigeneration systems. In Proc. of 6th World Energy System Conf., Turin, Italy, 2006.

Damoiseaux, A., Jokić, A., Lazar, M., Alessio, A., van den Bosch, P., Hiskens, I., and A., B. (2008). Assessment of decentralized model predictive control techniques for power networks. In PSCC 2008. Glasgow, Scotland.

Ebenbauer, C., Raff, T., and Allgöwer, F. (2009). Dissipation inequalities in systems theory: An introduction and recent results. In R. Jeltsch and G. Wanner (ed.), Invited Lectures of the International Congress on Industrial and Applied Mathematics 2007, 23-42.

EcoGrid (2009). Steps toward a danish power system with $50 \%$ wind energy. ECOGRID WP4: New measures for integration of large-scale renewable energy, 219.

ENTSO-E (2009). Operation Handbook. URL www.entsoe.eu/resources/publications/ entso-e/operation-handbook/.

EPEX (2010). European Power Exchange (EPEX). URL www . epexspot.com.

Galus, M.D., La Fauci, R., and Andersson, G. (2010). Investigating PHEV wind balancing capabilities using heuristics and model predictive control. In IEEE Power and Energy
Society (PES) General Meeting. Minneapolis, Minnesota, USA.

Gill, P.E., Murray, W., and Saunders, M.A. (2002). SNOPT: An SQP algorithm for large-scale constrained optimization. SIAM Journal on Optimisation, 12(4), 979-1006.

Heussen, K., Koch, S., Ulbig, A., and Andersson, G. (2010). Energy Storage in Power System Operation: The Power Nodes Modeling Framework. Presented at the IEEE PES Conference on Innovative Smart Grid Technologies Europe, Gothenburg, Sweden, 2010.

Jadbabaie, A. (2000). Nonlinear receding horizon control: a control lyapunov function approach. Ph.D. Thesis, CDS Caltech.

Mayne, D., Rawlings, J., Rao, C., and Scokaert, P. (2000). Constrained model predictive control: Stability and optimality. Automatica, 36(6):789-814.

Morari, M. and Lee, J. (1999). Model predictive control: Past, present and future. Computers and Chemical Engineering, $25,667-682$.

Negenborn, R., Beccuti, A., Demiray, T., Leirens, S., Damm, G., De Schutter, B., and Morari, M. (2007). Supervisory hybrid model predictive control for voltage stability of power networks. In American Control Conference. NYC, USA.

Oudalov, A., Chartouni, D., and Ohler, C. (2007). Optimizing a battery energy storage system for primary frequency control. IEEE Transactions on Power Systems, 22(3), 1259-1266.

Primbs, J.A. (1999). Nonlinear optimal control: A receding horizon approach. Ph.D. Thesis, CDS Caltech.

Quinn, C., Zimmerle, D., and Bradley, T.H. (2009). The effect of communication architecture on the availability, reliability, and economics of plug-in hybrid electric vehicle-to-grid ancillary services. Journal of Power Sources, In Press, Corrected Proof.

Sioshansi, R. (2010). Welfare impacts of electricity storage and the implications of ownership structure. The Energy Journal, 31(2), 173-198.

The Mathworks (2008). Optimization Toolbox User's Guide. URL www . mathworks. com.

Ulbig, A., Galus, M.D., Chatzivasileiadis, S., and Andersson, G. (2010). General Frequency Control with Aggregated Control Reserve Capacity from Time-Varying Sources: The Case of PHEVs. In IREP Symposium 2010 - Bulk Power System Dynamics and Control - VIII. Buzios, RJ, Brazil.

Voller, S., Al-Awaad, A.R., and Verstege, J.F. (2009). Wind farms with energy storages integrated at the control power market. In Integration of Wide-Scale Renewable Resources Into the Power Delivery System, 2009 CIGRE/IEEE PES Joint Symposium.

Weissbach, T. and Welfonder, E. (2009). High Frequency Deviations within the European Power System - Origins and Proposals for Improvement. In Invited paper in ELECTRA, vol. 242, p. 9-15. CIGRE.

Xie, L. and Ilić, M.D. (2009). Model predictive economic/environmental dispatch of power systems with intermittent resources. In Power and Energy Society (PES) General Meeting. Calgary, Canada.

Ying, C.M., Voorakaranam, S., and Joseph, B. (1999). Analysis and performance of the LP-MPC and QP-MPC cascade control system. In AIChE Journal, July 1999, Vol.45, No.7. 